\title{
Continuous-Time Tracking Filters for the Coordinated Turn Model
}

\author{
Bertil Ekstrand*
}

Ekelundsvägen 22, S-42942 Särö, Sweden

\begin{abstract}
Continuous-time tracking filters based on the coordinated turn model are discussed. By a coordinate transformation, the four-state filter design is reduced to design of two-state filters. An observer approach is applied, including pole placement and transfer function analysis. Deterministic steady-state errors for a circular turn are obtained as analytical expressions in target acceleration, filter bandwidth, and the actual and assumed turn rates. It is seen that no errors are obtained if the turn rate is known. If it is unknown, performance could still be better than with independent filters, if the assumed turn rate is not too erroneous. Even a first order transfer function can be formulated from which the steady-state errors are easily obtained. An interpretation in terms basic two-state filters is given of filters based on the coordinate turn model. Furthermore, the Kalman filter for a stochastic coordinated turn model is given in closed form and compared to the observers.
\end{abstract}

\section{INTRODUCTION}

Circular maneuvering is a basic case for target tracking. It is the common way to maneuver for civilian aircraft and is relevant also for evasive maneuvers. The tracking error for a circle is an important performance measure in practice and a bound on this error is a natural part of the requirements. The tracking literature on maneuvering targets is extensive and is dominated by different stochastic approaches, see [1-3] and further references therein. Performance is typically expressed as numerical Root-Mean-Square (RMS) errors obtained by stochastic Monte-Carlo simulations. Investigations based on observers and transfer functions are rare, although this approach includes requirements in a natural way and gives further insights into design and performance, as is well-known in control and other signal processing fields.

Using transfer and frequency functions, circular steadystate errors are studied in [4] for independent filters in the two Cartesian directions. A continuous-time two-state observer and the discrete-time $\alpha-\beta$ update Kalman filter are discussed. Radial and angular errors are obtained as analytical expressions in target acceleration and filter bandwidth. A new bandwidth interpretation of the tracking index is obtained, as well as a rule-of-thumb for an upper bound of this index. In [5] several topics for the prediction $\alpha-\beta-\gamma$ filter are discussed using transfer functions. The circular steadystate error for independent filters is also given and used in a particular optimization setting.

If independent filters are used, the coupling between the directions during a circle maneuver is neglected and steadystate errors are obtained. In the coordinated turn model, the CT model for short, this coupling is taken into account $[1,3]$. An example is given in [1, section 11.7] using the interacting multiple model (IMM) approach where also the CT model is

*Address correspondence to this author at the Ekelundsvägen 22, S-42942 Särö, Sweden; E-mail: bertilekstrand@telia.com included. Performance is evaluated in terms of numerical RMS errors obtained by simulations in a stochastic framework.

In the present paper tracking filter design based on the CT model is discussed. The continuous-time case is considered and an observer approach is applied, including transfer functions and pole placement. By a coordinate transformation, used in multivariable control systems [6], the four-state design is simplified to two-state cases. Using frequency functions, analytical expressions are obtained for the steadystate radial and angular errors in terms of target acceleration, filter bandwidth, and the actual and assumed turn rates. These expressions are useful for design, performance evaluation, and requirement formulations. No steady-state errors are obtained if the turn rate is known and the errors for an incorrect assumption of the turn rate are easily seen. It turns out that the steady-state performance can even be obtained from a simple first-order transfer function. An illustrative interpretation of CT-model filters is given in terms of basic second order filters. Furthermore, the Riccati equation for a stochastic CT model is solved in closed form and the associated Kalman filter is compared to the observer case.

Although discrete-time filters are used in practice, the continuous-time case is of interest. Methods and results are displayed more clearly in continuous-time. No sampling interval needs to be considered and the transfer functions are easier to interpret. Furthermore, the CT-model Kalman filter (of order four) can be solved in closed form. However, the order reduction coordinate transformation and the observer approach can be applied to the discrete-time case, of course, and so the methods and filter properties discussed have discrete-time counterparts. Comparisons between continuous and discrete-time tracking filters are further illustrated in [4].

If the turn rate is unknown, estimation of the turn rate parameter can be tried during the maneuver, as in the example in [1]. Although this is not addressed, the paper is of interest also for this case. The steady-state errors for an 
incorrect model turn rate are given, which should give some insights into the errors for an incorrect turn rate estimate, and so, into the question of what could be gained by turn rate estimation. The coordinate transformation method is of interest for the estimation case as well.

In the paper deterministic steady-state errors are discussed. Stochastic errors are obtained from measurement inaccuracies, often modeled reasonably well as white noise. As the filters are linear and time-invariant, deterministic and noise inputs can be treated separately and the output from a noise input can be superimposed on the deterministic output. The influence of white noise input to linear systems is a well-known topic [7]. In the present case this is further simplified as it is reduced to second order filters. In view of this, noise questions are not addressed, although some further comments are given in section 5 .

The CT model is introduced in section 2, observers are discussed in section 3, and steady-state errors are derived in section 4. The Kalman filter is given in section 5, as well as some remarks on the Kalman versus observer approaches, while observer polynomials and derivation of the Kalman filter can be found in Appendix.

\section{THE CT MODEL}

Circular motion of a target with constant speed in a horizontal plane is illustrated in Fig. (1). A Cartesian coordinate system $\xi, \eta$ is introduced, where the center of the circle is at the origin. Consider the situation where the position is measured by a sensor stationed at the origin, usually radar for the civilian aircraft case. In practice a sensor uses polar coordinates and it is assumed that polar-to-Cartesian conversion has been carried out. The measurements of the position $\xi, \eta$ are inputs to a filtering process, which gives estimates $\hat{\xi}, \hat{\eta}$ of the position. If the estimates are erroneous, the estimated position is different from the true one, as indicated in Fig. (1).

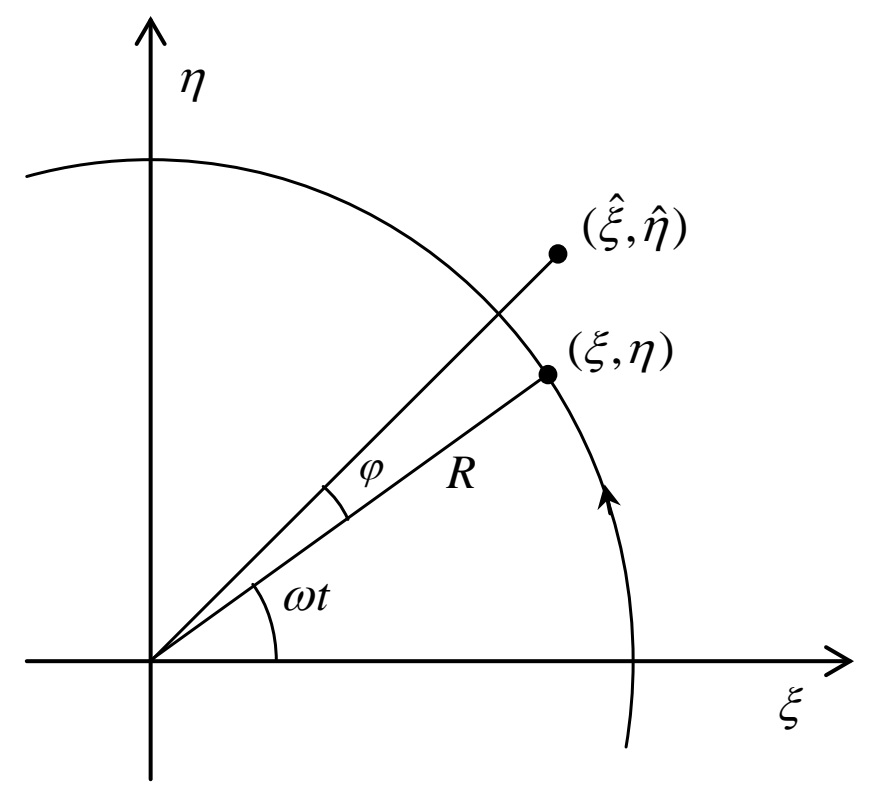

Fig. (1). Circular tracking.
Introduce the state vector

$x=\left[\begin{array}{cccc}\xi & \dot{\xi} & \eta & \dot{\eta}\end{array}\right]^{T} \triangleq\left[\begin{array}{llll}x_{1} & x_{2} & x_{3} & x_{4}\end{array}\right]^{T}$

and the measurement vector

$y=\left[\begin{array}{ll}y_{1} & y_{2}\end{array}\right]^{T}$

The CT model is $[1,3]$

$$
\begin{aligned}
& \dot{x}(t)=A x(t) \\
& y(t)=C x(t)
\end{aligned}
$$

where

$$
\begin{aligned}
A & =\left[\begin{array}{cccc}
0 & 1 & 0 & 0 \\
0 & 0 & 0 & -\omega \\
0 & 0 & 0 & 1 \\
0 & \omega & 0 & 0
\end{array}\right] \\
C & =\left[\begin{array}{lllc}
1 & 0 & 0 & 0 \\
0 & 0 & 1 & 0
\end{array}\right]
\end{aligned}
$$

and $\omega$ is the constant turn rate. (3a) is the kinematic equations for circular motion and $\omega$ implies a coupling between the two directions. The components of $y$ are the measurements of the position coordinates $\xi$ and $\eta$. For a circular turn of radius $R$, the measurements at time $t$ are

$$
\begin{aligned}
& y_{1}(t)=R \cos \tilde{\omega} t \\
& y_{2}(t)=R \sin \tilde{\omega} t
\end{aligned}
$$

where $\tilde{\omega}$ is the actual turn rate. It is assumed that the initial position is $(\xi, \eta)=(R, 0)$, but for steady-state performance the initial position is not significant. The same steady-state will be reached if the tracking starts at some other point of the circle, and (6) is used for simplicity.

The actual turn rate $\tilde{\omega}$ should be used for the model parameter $\omega$, that is, the idea is to take $\omega=\tilde{\omega}$ in the model. However, the actual turn rate is usually unknown, or known only within a certain range of accuracy. To take this uncertainty into account, the two notations $\omega$ and $\tilde{\omega}$ are used for the assumed and actual turn rate respectively.

If the sensor (and by that the origin) is not at the center of the maneuver circle, the coordinates of the circle center are inputs to the tracking filters as well, and a constant is then added to each of the inputs (6). However, as for the filters studied here, and as should be the case, tracking filters usually don't give steady-state errors for a constant input, that is, the constant center of the circle does not contribute to these errors. Steady-state errors are then obtained only from the circular inputs (6).

\section{CT MODEL OBSERVERS}

\section{General Observer}

The observer of the CT model (3) is

$\dot{\hat{x}}(t)=A \hat{x}(t)+K(y(t)-C \hat{x}(t))$ 
where $K$ is the constant filter gain, obtained by any method that will give a required performance. This observer has the same structure as the Kalman filter. That is, (7) is actually a Kalman filter if the gain is determined from the Riccati equation based on white noise disturbances added in the usual way in the model. However, as usual for observer design, no disturbances are assumed in the model. Denote the gain components as

$$
K=\left[\begin{array}{ll}
K_{1} & K_{5} \\
K_{2} & K_{6} \\
K_{3} & K_{7} \\
K_{4} & K_{8}
\end{array}\right]
$$

Laplace transformation gives $\hat{x}(s)=G(s) y(s)$ where $G(s)=(s I-A+K C)^{-1} K$. This transfer function has the polynomial structure

$$
G(s)=\frac{1}{D(s)}\left[\begin{array}{cc}
T_{1}(s) & T_{5}(s) \\
T_{2}(s) & T_{6}(s) \\
T_{3}(s) & T_{7}(s) \\
T_{4}(s) & T_{8}(s)
\end{array}\right]
$$

These polynomials, obtained after some straightforward calculations, are given in Appendix.

A basic property of the observer can be obtained immediately. The estimate of the position $x_{1}$ is

$$
\hat{x}_{1}(s)=\frac{T_{1}(s)}{D(s)} y_{1}(s)+\frac{T_{5}(s)}{D(s)} y_{2}(s)
$$

If $y_{1}$ and $y_{2}$ are constants, it is seen from the polynomials in Appendix (taking $s=0$ ) that in steady-state $\hat{x}_{1}=y_{1}$. Similarly for the other position, $\hat{x}_{3}=y_{2}$ in steady-state. Thus, for constant inputs the observer gives the correct positions in steady-state, and so the constant center of a maneuver circle does not contribute to the steady-state errors.

For pole placement design, the usual approach is to identify the characteristic polynomial $D(s)$ with a required polynomial and by that obtain equations for the gain factors $K_{i}$. In the present case the measurement vector $y$ has two components, which gives more complicated equations than with a single measurement component, in which case a system of linear equations is obtained. Furthermore, there are eight gain components, while the system is of order four, which means redundancy. Although a direct identification procedure is conceivable, at least by numerical methods, and could be simplified by the circular symmetry, a simplifying approach is possible, as follows.

\section{Two-State Observers}

In control terminology, (3) is a multivariable system as there are two measurement components (although no control inputs). By a coordinate transformation the design procedure can then be reduced to the single measurement case. To this end, as discussed in [6], introduce the new state vector

$$
z=T x=\left[\begin{array}{cccc}
0 & 1 & \omega & 0 \\
1 & 0 & 0 & 0 \\
-\omega & 0 & 0 & 1 \\
0 & 0 & 1 & 0
\end{array}\right] x
$$

Using that

$$
T^{-1}=\left[\begin{array}{rrrr}
0 & 1 & 0 & 0 \\
1 & 0 & 0 & -\omega \\
0 & 0 & 0 & 1 \\
0 & \omega & 1 & 0
\end{array}\right]
$$

the system equations (3) are transformed to

$$
\begin{aligned}
& \dot{z}(t)=T A T^{-1} z(t)=\left[\begin{array}{cccc}
0 & 0 & 0 & 0 \\
1 & 0 & 0 & -\omega \\
0 & 0 & 0 & 0 \\
0 & \omega & 1 & 0
\end{array}\right] z(t) \\
& y(t)=C T^{-1} z(t)=\left[\begin{array}{llll}
0 & 1 & 0 & 0 \\
0 & 0 & 0 & 1
\end{array}\right] z(t)
\end{aligned}
$$

which are in multivariable observable companion form [6]. Introduce the notations

$z=\left[\begin{array}{llll}z_{1} & z_{2} & z_{3} & z_{4}\end{array}\right]^{T}$

As $y_{1}=z_{2}$ and $y_{2}=z_{4}$, the system equations (13) can be written as

$$
\begin{aligned}
& {\left[\begin{array}{l}
\dot{z}_{1}(t) \\
\dot{z}_{2}(t)
\end{array}\right]=\left[\begin{array}{ll}
0 & 0 \\
1 & 0
\end{array}\right]\left[\begin{array}{l}
z_{1}(t) \\
z_{2}(t)
\end{array}\right]+\left[\begin{array}{c}
0 \\
-\omega
\end{array}\right] y_{2}(t)} \\
& y_{1}(t)=\left[\begin{array}{ll}
0 & 1
\end{array}\right]\left[\begin{array}{l}
z_{1}(t) \\
z_{2}(t)
\end{array}\right] \\
& {\left[\begin{array}{l}
\dot{z}_{3}(t) \\
\dot{z}_{4}(t)
\end{array}\right]=\left[\begin{array}{ll}
0 & 0 \\
1 & 0
\end{array}\right]\left[\begin{array}{l}
z_{3}(t) \\
z_{4}(t)
\end{array}\right]+\left[\begin{array}{c}
0 \\
\omega
\end{array}\right] y_{1}(t)} \\
& y_{2}(t)=\left[\begin{array}{ll}
0 & 1
\end{array}\right]\left[\begin{array}{l}
z_{3}(t) \\
z_{4}(t)
\end{array}\right]
\end{aligned}
$$

Two separate single-input/single-output systems of the same two-state structure are obtained, where $y_{1}$ and $y_{2}$, besides being measurements, also play the role of inputs. The observer of (15) is

$$
\begin{gathered}
{\left[\begin{array}{l}
\dot{\hat{z}}_{1}(t) \\
\dot{\hat{z}}_{2}(t)
\end{array}\right]=\left[\begin{array}{ll}
0 & 0 \\
1 & 0
\end{array}\right]\left[\begin{array}{l}
\hat{z}_{1}(t) \\
\hat{z}_{2}(t)
\end{array}\right]+\left[\begin{array}{c}
0 \\
-\omega
\end{array}\right] y_{2}(t)} \\
+\left[\begin{array}{l}
L_{1} \\
L_{2}
\end{array}\right]\left(y_{1}(t)-\left[\begin{array}{ll}
0 & 1
\end{array}\right]\left[\begin{array}{l}
\hat{z}_{1}(t) \\
\hat{z}_{2}(t)
\end{array}\right]\right) \\
=\left[\begin{array}{cc}
0 & -L_{1} \\
1 & -L_{2}
\end{array}\right]\left[\begin{array}{l}
\hat{z}_{1}(t) \\
\hat{z}_{2}(t)
\end{array}\right]+\left[\begin{array}{cc}
L_{1} & 0 \\
L_{2} & -\omega
\end{array}\right]\left[\begin{array}{l}
y_{1}(t) \\
y_{2}(t)
\end{array}\right]
\end{gathered}
$$

where $L_{1}, L_{2}$ are gain components to be determined. Laplace transformation gives 
$\left[\begin{array}{l}\hat{z}_{1}(s) \\ \hat{z}_{2}(s)\end{array}\right]=\frac{1}{s^{2}+L_{2} s+L_{1}}\left[\begin{array}{cc}L_{1} s & \omega L_{1} \\ L_{2} s+L_{1} & -\omega s\end{array}\right]\left[\begin{array}{l}y_{1}(s) \\ y_{2}(s)\end{array}\right]$

The corresponding observer of (16) is

$$
\begin{aligned}
& {\left[\begin{array}{l}
\dot{\hat{z}}_{3}(t) \\
\dot{\hat{z}}_{4}(t)
\end{array}\right]=\left[\begin{array}{cc}
0 & -L_{3} \\
1 & -L_{4}
\end{array}\right]\left[\begin{array}{l}
\hat{z}_{3}(t) \\
\hat{z}_{4}(t)
\end{array}\right]+\left[\begin{array}{cc}
0 & L_{3} \\
\omega & L_{4}
\end{array}\right]\left[\begin{array}{l}
y_{1}(t) \\
y_{2}(t)
\end{array}\right]} \\
& {\left[\begin{array}{l}
\hat{z}_{3}(s) \\
\hat{z}_{4}(s)
\end{array}\right]=\frac{1}{s^{2}+L_{4} s+L_{3}}\left[\begin{array}{cc}
-\omega L_{3} & L_{3} s \\
\omega s & s L_{4}+L_{3}
\end{array}\right]\left[\begin{array}{l}
y_{1}(s) \\
y_{2}(s)
\end{array}\right]}
\end{aligned}
$$

where $L_{3}, L_{4}$ are the gain components.

Thus, two observers of similar structure are obtained, each of which has two states and the measurement components as inputs. The advantage of this approach is obvious. Pole placement is immediate and for analysis the transfer functions (18) and (20) can be used. Clearly, a considerable simplification has been achieved by the transformation (11).

In the models (15) and (16) the turn rate parameter $\omega$ enters only in the input vectors associated with $y_{1}$ and $y_{2}$. The characteristic polynomials of the observers will then be independent of $\omega$. As is seen from the transfer functions (18) and (20), these polynomials depend only on the design parameters $L_{1}, L_{2}$ and $L_{3}, L_{4}$ respectively, and consequently, so do the poles.

\section{Four-State Observer}

The two-state observers give $\hat{z}$, and $\hat{x}$ can then be obtained by the inverse transformation $\hat{x}=T-1 \hat{z}$. Thus, the basic state vector $\hat{x}$ is obtained from the two-state observers. $\hat{x}$ can also be viewed as obtained directly from a four-state observer. Combining (17) and (19) gives

$$
\dot{\hat{z}}(t)=\tilde{A} \hat{z}(t)+L y(t)
$$

where

$$
\tilde{A}=\left[\begin{array}{cccc}
0 & -L_{1} & 0 & 0 \\
1 & -L_{2} & 0 & 0 \\
0 & 0 & 0 & -L_{3} \\
0 & 0 & 1 & -L_{4}
\end{array}\right] ; L=\left[\begin{array}{cc}
L_{1} & 0 \\
L_{2} & -\omega \\
0 & L_{3} \\
\omega & L_{4}
\end{array}\right]
$$

This is just another way to write the two-state observers. Multiplying (21) by $T^{-1}$, using that

$\tilde{A}=T A T^{-1}-L C T^{-1}$

yields the four-state observer

$$
\dot{\hat{x}}(t)=(A-K C) \hat{x}(t)+K y(t)
$$

where

$$
K=T^{-1} L=\left[\begin{array}{cc}
L_{2} & -\omega \\
L_{1}-\omega^{2} & -\omega L_{4} \\
\omega & L_{4} \\
\omega L_{2} & L_{3}-\omega^{2}
\end{array}\right]
$$

Input/output transfer functions for state-space representations of linear systems are not changed by coordinate transformations. In particular, the characteristic equation does not change. Using (24) in (54) gives $D(s)=\left(s^{2}+L_{2} s+L_{1}\right)\left(s^{2}+L_{4} s+L_{3}\right)$. That is, the poles of the four-state observer are the same as for the two-state observers. Design of (23) can then be carried out by design of the two-state observers. In this way the original observer (7) can be designed using (24) as the gain (8).

Considering the circular symmetry, and for simplicity, there is no reason to design the two-state observers differently. It is natural to take $L_{1}=L_{3}$ and $L_{2}=L_{4}$, that is, to choose an identical design for the two-state observers. It is convenient for design to write the characteristic polynomial as $s^{2}+2 \varsigma \omega_{0} s+\omega_{0}^{2}$, using second order standard notations. Thus, an "identity design" is obtained by taking

$L_{1}=L_{3}=\omega_{\mathrm{o}}^{2}$

$L_{2}=L_{4}=2 \varsigma \omega_{\mathrm{o}}$

To get good dynamical behavior, choice of the damping factor is normally restricted to a reasonable interval, for example $0.5<\zeta<1$, where $\zeta=0.7$ is commonly used. Thus, in practice $\zeta$ is essentially determined. Then the main significance of (25) is the possibility to obtain a certain $\omega_{0}$, which may be thought of as bandwidth. Requirements can often be formulated in terms of $\omega_{0}$. As will be seen below, this is the case for steady-state circular errors. Response time is another important performance parameter related to $\omega_{0}$, even by simple rules of thumb in basic cases. Clearly, $\omega_{\mathrm{o}}$ is a convenient design parameter by which design to meet requirements can be done.

By inserting the gain components (24) in the expressions (54) - (62) in Appendix, the transfer functions of the observer (23), as defined from $\hat{x}(s)=G(s) y(s)$, are obtained. Using the parameters $L_{1}$ and $L_{2}$ the identity design (25) yields

$G(s)=\frac{1}{s^{2}+L_{2} s+L_{1}}\left[\begin{array}{cc}T_{1}(s) & T_{5}(s) \\ T_{2}(s) & T_{6}(s) \\ T_{3}(s) & T_{7}(s) \\ T_{4}(s) & T_{8}(s)\end{array}\right]$

where

$$
\begin{aligned}
& T_{1}(s)=T_{7}(s)=L_{2} s+L_{1} \\
& T_{2}(s)=T_{8}(s)=\left(L_{1}-\omega^{2}\right) s \\
& T_{3}(s)=-T_{5}(s)=\omega s \\
& T_{4}(s)=-T_{6}(s)=L_{2} \omega s
\end{aligned}
$$

The transfer function (26), as well as the gain (24), has a symmetric structure. 


\section{An Interpretation}

An illustrative interpretation of the $\mathrm{CT}$ model observers is possible. From (26) the estimate of the position $x_{1}$ is

$$
\begin{aligned}
\hat{x}_{1}(s) & =\frac{L_{2} s+L_{1}}{s^{2}+L_{2} s+L_{1}} y_{1}(s)-\frac{\omega s}{s^{2}+L_{2} s+L_{1}} y_{2}(s) \\
& \triangleq F_{1}(s) y_{1}(s)-F_{2}(s) y_{2}(s)
\end{aligned}
$$

where the transfer function notations $F_{1}$ and $F_{2}$ have been introduced. It is composed of two parts: the output from $F_{1}$ driven by the input $y_{1}$, and the output from $F_{2}$ driven by $y_{2}$. $F_{1}$ is independent of $\omega$. In fact, $F_{1}$ is the basic second order filter obtained for the independent filter case, as discussed in [4]. The turn rate $\omega$ enters into $F_{2}$, and only at one point as a direct transfer function "gain".

It is reasonable for simplicity to start a design of tracking filters for circular maneuvers by considering identical independent filters of second order, and so obtain the filter $F_{1}$ in both directions. This is even more natural if such filters are used for straight-line tracking as is often the case. However, steady-state errors will then be obtained for circular maneuvers. These errors can be reduced to any required value by increasing the filter bandwidth [4], but if the requirement is high this could be inappropriate in practice. For example, it will increase the stochastic errors obtained from the measurement noise. If a CT model observer is introduced, it has the structure (31) for $\hat{x}_{1}$ and can be regarded as an attempt to improve the basic estimate obtained from $y_{1}$ and $F_{1}$, by using also the input $y_{2}$ via the transfer function $F_{2}$. A corresponding structure is obtained for $\hat{x}_{3}$.

Thus, the CT model observer can be interpreted as a way to complement the basic independent filter in one direction, by a particular use of the information about the position in the other direction. Whether this will actually be successful depends on how well the turn rate is known, as will now be discussed.

\section{STEADY-STATE ERRORS}

As the inputs (6) are sin and cos functions, it is clear that filter frequency functions should be useful to gain insights into steady-state circular errors for linear time-invariant tracking filters. Another useful fact is that the inputs are related, more exactly $\dot{y}_{2}(t)=\tilde{\omega} y_{1}(t)$, or by Laplace transformation

$s y_{2}(s)=\tilde{\omega} y_{1}(s)$

Using this input relationship and the transfer function (26) yields

$$
\begin{aligned}
& \frac{\hat{x}_{1}(s)}{y_{1}(s)}=\frac{L_{2} s+L_{1}-\omega \tilde{\omega}}{s^{2}+L_{2} s+L_{1}} \stackrel{\Delta}{=} G_{1}(s) \\
& \frac{\hat{x}_{3}(s)}{y_{2}(s)}=\frac{\frac{\omega}{\tilde{\omega}} s^{2}+L_{2} s+L_{1}}{s^{2}+L_{2} s+L_{1}} \stackrel{\Delta}{=} G_{2}(s)
\end{aligned}
$$

Thus, the observer output $\hat{x}_{1}$, using both measurements, is the same as the output from $G_{1}(s)$ driven only by the input $y_{1}$. Similarly, $\hat{x}_{3}$ is obtained from $G_{2}(s)$ via the input $y_{2}$. Now

$G_{1}(i \tilde{\omega})=G_{2}(i \tilde{\omega})=\frac{\omega_{\mathrm{o}}^{2}-\omega \tilde{\omega}+i 2 \varsigma \omega_{\mathrm{o}} \tilde{\omega}}{\omega_{\mathrm{o}}^{2}-\tilde{\omega}^{2}+i 2 \varsigma \omega_{\mathrm{o}} \tilde{\omega}} \stackrel{\Delta}{=} W$

That is, the two frequency functions are equal for the actual turn rate frequency $\tilde{\omega}$. The notation $W$ has been introduced for this frequency function value. As the inputs $y_{1}$ and $y_{2}$ are the cos and sin functions (6), the steady-state outputs are

$\hat{x}_{1}(t)=R|W| \cos (\tilde{\omega} t+\varphi)$
$\hat{x}_{3}(t)=R|W| \sin (\tilde{\omega} t+\varphi)$

where $\varphi=R \arg W$. Thus, according to the filter the target moves along a circle of radius $R|W|$ and has the angular separation $\varphi$ from the actual position. Errors are obtained if $W \neq 1$. As in [4], introduce

Radial error: $\quad e_{r}=R(|W|-1)$

Angular error: $\quad e_{\varphi}=R \varphi=R \arg W$

Both these errors are length displacement errors, $e_{r}$ in the radial direction and $e_{\varphi}$ along the circle. Beside the magnitudes, these errors contribute further illustrative information as well: it is seen that the filtering gives a circle outside the actual one if the radial error is positive and that the position is delayed if the angular error is negative. The total error is easily obtained from the separate errors.

From (35) it is seen that $W=1$ if $\omega=\tilde{\omega}$, which means zero errors (37), (38). That is, if the correct turn rate is used in the model, no steady-state errors are obtained. If $\omega \neq \tilde{\omega}$ there will be errors. For convenience, introduce

$d=\frac{\omega}{\omega_{\mathrm{o}}} ; \quad h=\frac{\tilde{\omega}}{\omega_{\mathrm{o}}}$

$d$ is the ratio between the model turn rate parameter $\omega$ and the filter parameter $\omega_{0}$, while $h$ is the corresponding ratio using the actual turn rate $\tilde{\omega}$. After some calculations

$|W|=\sqrt{1+\frac{2 h(h-d)(1-h(h+d) / 2)}{1+h^{4}+2 h^{2}\left(2 \varsigma^{2}-1\right)}}$

The radial error (37) can now be obtained. A useful approximation is possible, though. In practice $2 \zeta^{2} \approx 1$ and $d<<$ $1, h<<1$. Then

$|W| \approx \sqrt{1+2 h(h-d)} \approx 1+h(h-d)$

and the radial error becomes

$e_{r} \approx R\left(\frac{\tilde{\omega}}{\omega_{0}}\right)^{2}\left(1-\frac{\omega}{\tilde{\omega}}\right)$ 
It is interesting to compare with the corresponding independent filters discussed in [4], in which case the radial error is (approximately) $R\left(\widetilde{\omega} / \omega_{\mathrm{o}}\right)^{2}$. For the CT case (42) there is a further factor $1-\omega / \tilde{\omega}$. Again it is seen that if the correct turn rate $\omega=\tilde{\omega}$ is used the error is zero, in contrast to independent filters. However, for an unknown turn rate, the CT case could still be better than independent filters if the assumed value is reasonably close to the actual value. In any case, the error can be reduced by increasing the filter parameter $\omega_{0}$, that is, by increasing the filter bandwidth.

For the angular error

$$
\begin{aligned}
\arg W & =-\arctan \frac{2 \varsigma h^{2}(h-d)}{(1-d h)\left(1-h^{2}\right)+4 \varsigma^{2} h^{2}} \\
& \approx-2 \varsigma h^{2}(h-d)
\end{aligned}
$$

which gives

$e_{\varphi} \approx-2 \varsigma \frac{\tilde{\omega}}{\omega_{0}} e_{r}$

This is the same expression as for the independent filters in [4]. The angular error is usually much smaller than the radial error since $\widetilde{\omega} / \omega_{0}$ is small. That is, the steady-state error is mainly in the radial direction.

A target turn is often characterized by its acceleration, normally expressed in units of $g$. This can be done by using the kinematic relations $V=R \widetilde{\omega}$ and $a_{r}=V^{2} / R$, where $V$ is the target velocity and $a_{r}$ the (radial) acceleration. The radial error (42) can then be expressed as

$e_{r} \approx \frac{a_{r}}{\omega_{\mathrm{o}}^{2}}\left(1-\frac{\omega}{\widetilde{\omega}}\right)$

The velocity $V$ and the radius $R$ are not involved explicitly; for a given ratio $\omega / \widetilde{\omega}$ they influence the error only via $a_{r}$.

No errors are obtained for $\omega=\widetilde{\omega}$. This is a fact irrespective of the particular identical design, that is, irrespective of $\omega_{0}$ and $\zeta$, as is seen from (35). From this point of view, there is no reason for small values of $d$ and $h$, as assumed to obtain the error approximations (42) and (44). However, as the exact turn rate is typically unknown, to keep the errors reasonably small in practice, the filter parameter $\omega_{0}$ has to be considerably higher than the turn rates $\omega$ and $\widetilde{\omega}$. Thus, in practice $d<<1$ and $h \ll<1$ as assumed.

It should be noted that (35) can be regarded as obtained from the frequency function of the transfer function

$F(s)=\frac{s+b}{s+c}$

where

$b=\frac{\omega_{\mathrm{o}}}{2 \varsigma}(1-h d)$ $c=\frac{\omega_{\mathrm{o}}}{2 \varsigma}\left(1-h^{2}\right)$

In fact, (35) is the frequency function value $F(i \widetilde{\omega})$ of (46) for the actual turn rate frequency $\widetilde{\omega}$. Thus, the steadystate circular errors can be obtained from the simple transfer function (46).

A further question of interest is how fast the steady-state will be reached. This is determined by the filter response time (or equivalently by $\omega_{0}$ ). As discussed in [4], since $\widetilde{\omega} / \omega_{0}$ is small in practice, the steady-state is normally reached after a minor part of the maneuver circle.

\section{KALMAN FILTER}

To obtain a Kalman filter, stochastic disturbances are introduced in the usual way in the model (3)

$$
\begin{aligned}
& \dot{x}(t)=A x(t)+v(t) \\
& y(t)=C x(t)+w(t)
\end{aligned}
$$

where as usual $v(t)$ and $w(t)$ are zero mean, white noises with covariance

$$
\begin{aligned}
& E\left[v(t) v(\tau)^{\prime}\right]=Q \delta(t-\tau) \\
& E\left[w(t) w(\tau)^{\prime}\right]=R \delta(t-\tau)
\end{aligned}
$$

Consider the choice

$Q=\left[\begin{array}{llll}0 & 0 & 0 & 0 \\ 0 & q & 0 & 0 \\ 0 & 0 & 0 & 0 \\ 0 & 0 & 0 & q\end{array}\right] ; R=\left[\begin{array}{ll}r & 0 \\ 0 & r\end{array}\right]$

$R$ implies that the two measurement components are independent and has the same accuracy. $Q$ may seem overly simplified, but the $2 \times 2$ identical block diagonal matrices of $Q$ are commonly used as the covariance in two-state continuous-time stochastic models, either to obtain continuous-time Kalman filters, or to obtain discrete-time models by sampling and by that discrete-time Kalman filters. The covariance $Q$ is an extension to the four-state case.

The CT-model part in the example in [1] is a four-state extension of the two-state so called direct discrete-time model, which is quite close to the model obtained by sampling the two-state continuous-time model mentioned above. In view of this, (48) - (50) can be considered as a stochastic continuous-time model, which essentially corresponds to the discrete-time CT-model part in [1].

The algebraic Riccati equation for the model (48) - (50) is solved in closed form in Appendix, which gives the gain

$$
K=\frac{1}{2}\left[\begin{array}{cc}
2 a & 0 \\
a^{2} & -a \omega \\
0 & 2 a \\
a \omega & a^{2}
\end{array}\right]
$$


$a=\sqrt{2} \sqrt{-\frac{\omega^{2}}{4}+\sqrt{\left(\frac{\omega^{2}}{4}\right)^{2}+\frac{q}{r}}}$

The steady-state Kalman filter is obtained by using this gain in the observer (23). The gain has a symmetric structure similar to the observer gain (24) for the identity design (25). By considering the Kalman filter polynomials in Appendix, it is seen that the gain (51) corresponds essentially to an observer gain with $a=\omega_{0} \sqrt{2}$ and $\varsigma=1 / \sqrt{2}$ since $\omega$ is small in practice. Consequently, as for $\omega_{0}$, the parameter $a$ may be thought of as bandwidth.

The transfer functions and the steady-state circular errors for this Kalman filter are given in Appendix. Expressed in radial acceleration, the radial error (86) is

$e_{r} \approx 2 \frac{a_{r}}{a^{2}}\left(1-\frac{\omega}{\tilde{\omega}}\right)$

This is the same expression as for the observer case (42) if $a=\omega_{\mathrm{o}} \sqrt{2}$. The angular error is slightly different from the observer case, but as for this case, this error is usually much smaller than the radial error. The radial error is the dominating part of the total error also for the Kalman filter.

\section{Further Remarks}

It should be noted that for the Kalman filter $q / r$ is a design parameter (in fact the only one), as this ratio determines the parameter $a$ and by that the gain. For example, $q / r$ can be chosen to keep the steady-state error (53) below a certain bound. If $r$ is considered as a model parameter (which is reasonable), then $q$ is the design parameter, which has to be chosen to give a required filter (if possible).

It can be argued that this is how noise parameters have to be conceived when the Kalman filter is used in tracking applications. As in other fields, certain filter requirements are always involved in practice, typically concerning bandwidth, response time, and steady-state errors, and the only way to fulfill the requirements for a given stochastic model structure is by choice of the noise parameters.

This view is supported by the fact that white process noise cannot be regarded as a reasonable maneuver model. Consider for example the (continuous- or discrete-time) $\alpha-\beta$ Kalman tracking filter, in which case the target acceleration is assumed to be white process noise [1]. However, as constant or nearly constant acceleration is used for maneuvering, it is certainly not white. The only reasonable way to interpret the acceleration process noise parameter is as a design parameter [8]. It should be noted that if coloured process noise is introduced instead, performance could deteriorate [9].

Furthermore, it should be noted that requirements are actually neglected in the stochastic approach to tracking. Consequently, design to meet requirements cannot be done. If requirements are taken into account (as should be the case of course), a stochastic design process will be quite complex, involving extensive stochastic simulations for different noise parameter values, in which case the noise parameters are used as design parameters anyhow. Clearly, an observer ap- proach, using pole placement and transfer functions as above, is more simple and efficient, and includes requirements in a natural way. As pointed out in the introduction, an output caused by measurement noise can be superimposed on the deterministic output. Analytical expressions for this are given in [7].

It should be noted that design is actually a notion that refers to activities and methods used to obtain a filter that fulfills the requirements. Without requirements there is no need for design, in fact any filter would do. In practice some requirements are always specified and needed.

Further aspects on these issues can be found in [4] and [8-10].

\section{CONCLUSIONS}

Continuous-time tracking filters based on the coordinated turn model are discussed. By a coordinate transformation the four-state filter design is transformed to design of two-state filters. Using filter frequency functions, the deterministic steady-state radial and angular errors for a circular turn are obtained as analytical expressions in target acceleration, filter bandwidth, and the actual and assumed turn rates. It is seen that no errors are obtained if the turn rate is known. If it is unknown, performance could still be better than with independent filters if the assumed turn rate is reasonably close to the actual value. Even a simple first order transfer function can be formulated from which the errors are obtained. An interpretation of CT-model filters is given in terms of basic second order filters. Furthermore, a Kalman filter for the coordinated turn model is solved in closed form and compared to the observers. It is argued that the noise parameters in the Kalman filter should be considered as a design parameters. The paper is observer and transfer function based and illustrates the benefits of this approach.

\section{APPENDIX}

\section{Transfer Function Polynomials}

Using the gain (8) in the observer (7) gives the following polynomials in the transfer function (9).

$$
\begin{aligned}
& D(s)=s^{4}+\left(K_{1}+K_{7}\right) s^{3}+\left(K_{2}+K_{8}+K_{1} K_{7}\right. \\
& \left.\quad-K_{3} K_{5}+\omega^{2}\right) s^{2}+\left[K_{1} K_{8}+K_{2} K_{7}-K_{3} K_{6}\right. \\
& \left.\quad-K_{4} K_{5}+\left(K_{1}+K_{7}\right) \omega^{2}+\left(K_{6}-K_{4}\right) \omega\right] s \\
& \quad+K_{2} K_{8}-K_{4} K_{6}+\left(K_{1} K_{7}-K_{3} K_{5}\right) \omega^{2} \\
& \quad+\left(K_{1} K_{6}+K_{3} K_{8}-K_{2} K_{5}-K_{4} K_{7}\right) \omega \\
& T_{1}(s)=K_{1} s^{3}+\left(K_{2}+K_{1} K_{7}-K_{3} K_{5}\right) s^{2}+\left(K_{1} K_{8}\right. \\
& \left.\quad+K_{2} K_{7}-K_{3} K_{6}-K_{4} K_{5}+K_{1} \omega^{2}-K_{4} \omega\right) s \\
& \quad+K_{2} K_{8}-K_{4} K_{6}+\left(K_{1} K_{7}-K_{3} K_{5}\right) \omega^{2} \\
& \quad+\left(K_{1} K_{6}+K_{3} K_{8}-K_{2} K_{5}-K_{4} K_{7}\right) \omega \\
& T_{2}(s)=s\left[K_{2} s^{2}+\left(K_{2} K_{7}-K_{3} K_{6}-K_{4} \omega\right) s+K_{2} K_{8}\right. \\
& \left.\quad-K_{4} K_{6}+\left(K_{3} K_{8}-K_{4} K_{7}\right) \omega\right] \\
& T_{3}(s)=s\left(K_{3} s^{2}+K_{4} s+K_{3} \omega^{2}+K_{2} \omega\right)
\end{aligned}
$$




$$
\begin{aligned}
T_{4}(s) & =s\left[K_{4} s^{2}+\left(K_{4} K_{7}-K_{3} K_{8}+K_{2} \omega\right) s\right. \\
& \left.+\left(K_{2} K_{7}-K_{3} K_{6}\right) \omega\right] \\
T_{5}(s) & =s\left(K_{5} s^{2}+K_{6} s+K_{5} \omega^{2}-K_{8} \omega\right) \\
T_{6}(s) & =s\left[K_{6} s^{2}+\left(K_{1} K_{6}-K_{2} K_{5}-K_{8} \omega\right) s\right. \\
& \left.+\left(K_{4} K_{5}-K_{1} K_{8}\right) \omega\right] \\
T_{7}(s) & =K_{7} s^{3}+\left(K_{8}+K_{1} K_{7}-K_{3} K_{5}\right) s^{2}+\left(K_{1} K_{8}\right. \\
& \left.+K_{2} K_{7}-K_{3} K_{6}-K_{4} K_{5}+K_{7} \omega^{2}+K_{6} \omega\right) s \\
& +K_{2} K_{8}-K_{4} K_{6}+\left(K_{1} K_{7}-K_{3} K_{5}\right) \omega^{2} \\
& +\left(K_{1} K_{6}+K_{3} K_{8}-K_{2} K_{5}-K_{4} K_{7}\right) \omega \\
T_{8}(s) & =s\left[K_{8} s^{2}+\left(K_{1} K_{8}-K_{4} K_{5}+K_{6} \omega\right) s\right. \\
& \left.+K_{2} K_{8}-K_{4} K_{6}+\left(K_{1} K_{6}-K_{2} K_{5}\right) \omega\right]
\end{aligned}
$$

\section{Riccati Equation}

The algebraic Riccati equation for the model (48), (49) is

$$
A P+P A^{T}-P C^{T} R^{-1} C P+Q=0
$$

Denote $P=\left[p_{i j}\right]$ and let $Q, R$ be given by (50). Using the fact that $P$ is symmetric, the component equations of (63) are

$$
\begin{aligned}
& \frac{1}{r}\left(p_{11}^{2}+p_{13}^{2}\right)-2 p_{12}=0 \\
& \frac{1}{r}\left(p_{11} p_{12}+p_{13} p_{23}\right)-p_{22}+\omega p_{14}=0 \\
& \frac{1}{r}\left(p_{11} p_{13}+p_{13} p_{33}\right)-p_{14}-p_{23}=0 \\
& \frac{1}{r}\left(p_{11} p_{14}+p_{13} p_{34}\right)-p_{24}-\omega p_{12}=0 \\
& \frac{1}{r}\left(p_{12}^{2}+p_{23}^{2}\right)+2 \omega p_{24}-q=0 \\
& \frac{1}{r}\left(p_{12} p_{13}+p_{23} p_{33}\right)-p_{24}+\omega p_{34}=0 \\
& \frac{1}{r}\left(p_{12} p_{14}+p_{23} p_{34}\right)-\omega\left(p_{22}-p_{44}\right)=0 \\
& \frac{1}{r}\left(p_{13}^{2}+p_{33}^{2}\right)-2 p_{34}=0 \\
& \frac{1}{r}\left(p_{13} p_{14}+p_{33} p_{34}\right)-p_{44}-\omega p_{23}=0 \\
& \frac{1}{r}\left(p_{14}^{2}+p_{34}^{2}\right)-2 \omega p_{24}-q=0
\end{aligned}
$$

These equations can be solved in the following way. Start by the assumptions $p_{11}=p_{33}$ and $p_{22}=p_{44}$, as is reasonable by symmetry. Then, (64) and (71) give $p_{12}=p_{34}$, which gives $p_{14}=-p_{23}$ using (70) (as from (64) $p_{12}>0$ ). Now (66) gives $p_{13}=0$ and it follows from (68) and (73) that $p_{24}=0$. From (64) $p_{11}^{2}=2 r p_{12}$ and using (67) it is found that $p_{14}=\omega p_{11} / 2$. Then (68) yields a second order equation for $p_{12}$, which has only one solution (as $p_{12}>0$ ), and finally $p_{44}$ is obtained via (72). Using the parameter

$$
a=\sqrt{2} \sqrt{-\frac{\omega^{2}}{4}+\sqrt{\left(\frac{\omega^{2}}{4}\right)^{2}+\frac{q}{r}}}
$$

the result of this procedure is

$$
\begin{aligned}
& p_{11}=p_{33}=a r \\
& p_{12}=p_{34}=\frac{1}{2} a^{2} r \\
& p_{13}=p_{24}=0 \\
& p_{14}=-p_{23}=\frac{1}{2} \operatorname{ar} \omega \\
& p_{22}=p_{44}=\frac{1}{2} \operatorname{ar}\left(a^{2}+\omega^{2}\right)
\end{aligned}
$$

These components satisfy (64) - (73) and it can be shown that they give a positive definite $P$. Thus, although the starting point was an assumption, (75) is in fact the (unique) solution to the algebraic Riccati equation (63). The Kalman gain $K=P C^{T} R^{-1}$ becomes

$$
K=\frac{1}{r}\left[\begin{array}{ll}
p_{11} & p_{13} \\
p_{12} & p_{23} \\
p_{13} & p_{33} \\
p_{14} & p_{34}
\end{array}\right]=\frac{1}{2}\left[\begin{array}{cc}
2 a & 0 \\
a^{2} & -a \omega \\
0 & 2 a \\
a \omega & a^{2}
\end{array}\right]
$$

\section{Kalman Filter Polynomials}

Inserting the Kalman gain (76) into the expression (54) yields the characteristic polynomial

$$
D(s)=\left(s^{2}+a s+\frac{a^{2}}{2}\right)^{2}+\omega^{2}\left(s+\frac{a}{2}\right)^{2}
$$

Due to the particular form of this polynomial, it is easy to solve the characteristic equation $D(s)=0$. It is a matter of solving second order equations and the result is

$$
s=-\frac{a}{2}\left[1 \pm i\left(\frac{\omega}{a} \pm \sqrt{1+\left(\frac{\omega}{a}\right)^{2}}\right)\right]
$$

Using the signs independently yields the four poles. For $\omega=0$ double poles are obtained in $s=-a(1 \pm i) / 2$, which by second order standard notations corresponds to $\omega_{0}=a / \sqrt{2}$ and $\varsigma=1 / \sqrt{2}$. The numerator polynomials (54) - (62) become 
$T_{1}(s)=T_{7}(s)=a\left(s+\frac{a}{2}\right)\left(s^{2}+a s+\frac{a^{2}}{2}+\frac{\omega^{2}}{2}\right)$

$T_{2}(s)=T_{8}(s)=\frac{a^{2}}{2} s\left[s^{2}+a s+\frac{a^{2}}{2}-\frac{\omega^{2}}{a}\left(s+\frac{a}{2}\right)\right]$

$T_{3}(s)=-T_{5}(s)=\frac{a \omega}{2} s(s+a)$

$T_{4}(s)=-T_{6}(s)=\frac{a \omega}{2} s(s+a)^{2}$

As for the observer case, several of the transfer function polynomials are the same.

\section{Kalman Filter Steady-State Errors}

The circular steady-state errors for the Kalman filter are obtained in the same way as for the observers. Using the input relation (32), the transfer functions corresponding to (33) and (34) are

$$
\begin{aligned}
& \frac{\hat{x}_{1}(s)}{y_{1}(s)}=\frac{1}{D(s)}\left[T_{1}(s)+\frac{\tilde{\omega}}{s} T_{5}(s)\right] \triangleq H_{1}(s) \\
& \frac{\hat{x}_{3}(s)}{y_{2}(s)}=\frac{1}{D(s)}\left[T_{3}(s) \frac{s}{\tilde{\omega}}+T_{7}(s)\right] \triangleq H_{2}(s)
\end{aligned}
$$

From the similarities of the polynomials in (79) and (81), it is seen that these two transfer functions have the same frequency function value for the actual turn rate frequency $\tilde{\omega}$, that is

$$
H_{1}(i \tilde{\omega})=H_{2}(i \tilde{\omega})
$$

As for the observers, this frequency function value determines the steady-state errors, which, after some calculations and some approximations based on the assumptions $\omega$ $<<a, \widetilde{\omega}<<a$, are found to be

$$
\begin{aligned}
& e_{r} \approx 2 R\left(\frac{\tilde{\omega}}{a}\right)^{2}\left(1-\frac{\omega}{\tilde{\omega}}\right) \\
& e_{\varphi} \approx-2 \frac{\tilde{\omega}}{a}\left(1-\frac{1}{2} \frac{\omega}{\tilde{\omega}}\right) e_{r}
\end{aligned}
$$

\section{REFERENCES}

[1] Y. Bar-Shalom, X.R. Li, and T. Kirubarajan, "Estimation with Applications to Tracking and Navigation”, New York: Wiley, 2001.

[2] Y. Bar-Shalom, and T.E. Fortmann, "Tracking and Data Association”, San Diego, CA: Academic Press, 1988.

[3] X.R. Li, and V.P. Jilkov, "Survey of maneuvering target tracking. Part I: dynamic models", IEEE Transactions on Aerospace and Electronic Systems, vol. 39(4), pp. 1333-1364, 2003.

[4] B. Ekstrand, "Steady-state circular errors for basic tracking filters", IEE Proceedings Control Theory \& Applications, vol. 153(4), pp. 413-419, 2006.

[5] D. Tenne, and T. Singh, "Characterizing performance of $\alpha-\beta-\gamma$ filters", IEEE Transactions on Aerospace and Electronic Systems, vol. 38(3), pp. 1072-1087, 2002.

[6] W.A. Wolovich, "Linear Multivariable Systems", Applied Mathematical Sciences, New York: Springer-Verlag, Vol. 11, 1974.

[7] K.J. Åström, Introduction to Stochastic Control Theory, New York: Academic Press, 1970.

[8] B. Ekstrand, "Poles and zeros of $\alpha-\beta$ and $\alpha-\beta-\gamma$ tracking filters", IEE Proceedings Control Theory \& Applications, vol. 148(5), pp. 370-376, 2001

[9] B. Ekstrand, "Design aspects of a continuous-time tracking filter", IEE Proceedings Control Theory \& Applications, vol. 151(1), pp. $1-5,2004$.

[10] B. Ekstrand, "Tracking Filters and Models for Seekern Applications", IEEE Transactions on Aerospace and Electronic System, vol. 37(3), pp. 965-977, 2001. 